compared to other mammals, allowing viruses to exist in bats without raising red flags with their immune system. Taking to the skies may have given bats the mean to cope with viruses that other mammals cannot.

https://doi.org/10.1038/s41684-018-0037-4

\section{BIOLOGICAL TECHNIQUES}

\section{Testing for torpor}

J. Exp. Biol. https://doi.org/10.1242/jeb.174508 (2018)

Hibernating mammals remain enigmatic in their ability to sustain themselves through extended bouts of torpor, during which their metabolic rates and body temperatures markedly decline. Knowing exactly when an animal enters and exits torpor has been difficult to determine, with most definitions relying on body temperature. But that measure is bit rough and might miss physiological and metabolic changes that occur before a temperature threshold is passed.

To overcome this limitation, researchers from the University of Western Ontario have developed a detection method that relies instead on heartbeat. They implanted thirteen-lined ground squirrels with small electrocardiogram telemeters and recorded their hibernation. The simple technique revealed that as the animals entered and exited torpor states, heart rate began to change well before body temperature. EPN

https://doi.org/10.1038/s41684-018-0038-3

\section{NEURAL CIRCUITS}

\section{On the hunt}

Nat. Neurosci. https://doi.org/10.1038/s41593-

018-0072-x (2018)

Animals appear to have an innate desire to interact with novel objects, and this is key to hunting or foraging. Researchers have not identified the neural networks that drive this desire, but the ventral periaqueductal gray (VPAG) area of the brain has long been suspected.

The medial preoptic area (MPA), found in the hypothalamus, sends projections to the VPAG and influences mating and maternal behavior. Researchers at KAIST in Daejeon, Korea used a genetic trick to show that MPA cells containing the $\alpha$ subunit of $\mathrm{Ca} 2+/$ calmodulin-dependent kinase II (CaMKII $\alpha)$ may be the responsible activators. The researchers confirmed the effect by engineering mice so that these cells would be activated by blue light. When the cells were illuminated, the mice engaged more in chasing objects.

https://doi.org/10.1038/s41684-018-0039-2

\section{GENETICS}

\section{The ageless bat}

Sci. Adv. 4, eaao0926 (2018)

Bats can live a long time, the longest of all mammals relative to their body size. That makes them unique models for understanding aging and the factors that contribute to a species' life span. Telomeres, protective nucleotide repeats found on the ends of chromosomes but shorten with every cell division, are thought to play a role. For the first time, a new study examines telomere length relative to the lifespans of four bat species sampled in the wild. In the shorter-lived species, telomere length declined with age, but no such trend was observed in bats of the Myotis genus, the current age record holders. These little bats, upon genetic comparison with other mammals, have enriched expression of genes involved in telomere maintenance and DNA repair. EPN

https://doi.org/10.1038/s41684-018-0040-9

Ellen P. Neff, Jim Kling

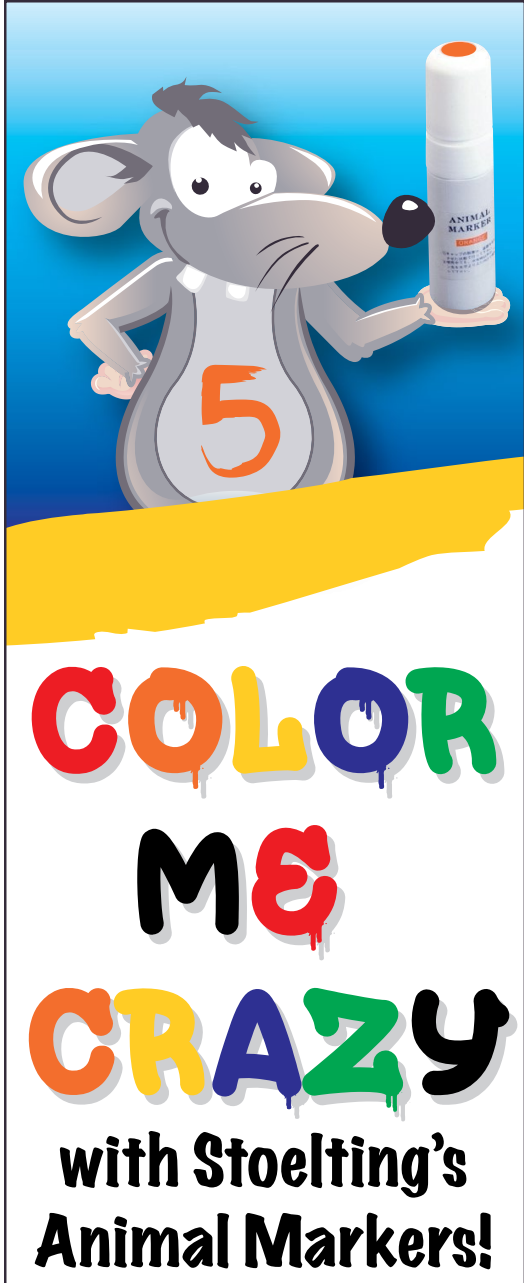

- Non-toxic

- Non-invasive

- Single marker can mark 300-500 mice or rats

- Colors remain for 6-12 weeks

- Available in Red, Orange, Yellow, Green, Blue and Black

- Standard and fine-tip markers available

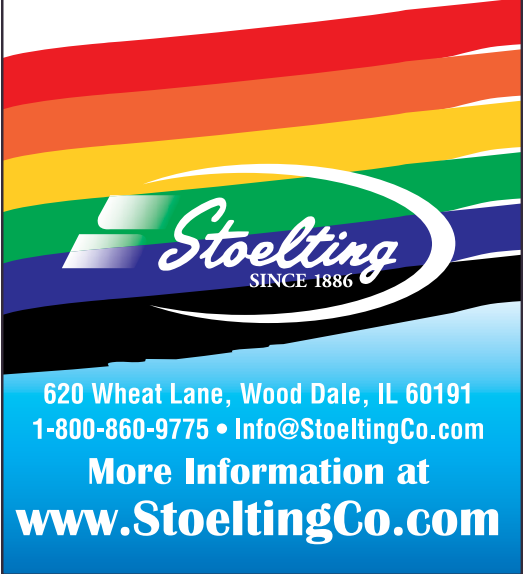

